\title{
FACTORIZATION AND REFLEXIVITY ON FOCK SPACES
}

\author{
Alvaro Arias and Gelu Popescu
}

\begin{abstract}
The framework of the paper is that of the full Fock space $\mathcal{F}^{2}\left(\mathcal{H}_{n}\right)$ and the Banach algebra $F^{\infty}$ which can be viewed as non-commutative analogues of the Hardy spaces $H^{2}$ and $H^{\infty}$ respectively.

An inner-outer factorization for any element in $\mathcal{F}^{2}\left(\mathcal{H}_{n}\right)$ as well as characterization of invertible elements in $F^{\infty}$ are obtained. We also give a complete characterization of invariant subspaces for the left creation operators $S_{1}, \cdots, S_{n}$ of $\mathcal{F}^{2}\left(\mathcal{H}_{n}\right)$. This enables us to show that every weakly (strongly) closed unital subalgebra of $\left\{\varphi\left(S_{1}, \cdots, S_{n}\right): \varphi \in F^{\infty}\right\}$ is reflexive, extending in this way the classical result of Sarason [S].

Some properties of inner and outer functions and many examples are also considered.
\end{abstract}

\section{INTRODUCTION}

Let $H^{p}, 1 \leq p \leq \infty$ be the classical Hardy spaces on the disk. $H^{2}$ is a Hilbert space with orthonormal basis $\left\{z^{n}\right\}_{n=0}^{\infty}$ and it is well known that if $\varphi \in H^{\infty}$, then $\|\varphi\|_{\infty}=\left\|M_{\varphi}\right\|$, where $M_{\varphi}: H^{2} \rightarrow H^{2}$ is the multiplication operator. Hence,

$$
\|\varphi\|_{\infty}=\sup \left\{\|\varphi p\|_{2}:\|p\|_{2} \leq 1 \text {, and } p \text { is a polynomial on } z\right\} .
$$

The set of polynomials on $z, \mathcal{P}(z)$, determine the Hardy spaces: $H^{2}$ is the closure of $\mathcal{P}(z)$ in the Hilbert space with orthonormal basis $\left\{z^{n}\right\}_{n=0}^{\infty}$. Once we have $H^{2}$, $H^{\infty}$ consists of all $\varphi \in H^{2}$ such that (1) is finite.

In [Po3], [Po4] the second author introduced and studied a "non-commutative" analogue of $H^{\infty}$. Let $\mathcal{P}$ be the set of polynomials in $n$ non-commutative indeterminates $e_{1}, \cdots, e_{n}$. To stress the non-commutativity of the product we use tensor notation; (i.e., $e_{1} \otimes e_{2}$ represents the product of $e_{1}$ and $e_{2}$ and is different from $\left.e_{2} \otimes e_{1}\right)$. A typical element of $\mathcal{P}$ looks like

$$
p=a_{0}+\sum_{k=1}^{m} \sum_{1 \leq i_{1}, \cdots, i_{k} \leq n} a_{i_{1} \cdots i_{k}} e_{i_{1}} \otimes \cdots \otimes e_{i_{k}},
$$


where $m \in \mathbb{N}$ and $a_{0}, a_{i_{1} \cdots i_{k}} \in \mathbb{C}$. Let $\mathcal{F}^{2}\left(\mathcal{H}_{n}\right)$ be the Hilbert space having the monomials as an orthonormal basis (i.e., 1 and the elements of the form $e_{i_{1}} \otimes \cdots \otimes$ $\left.e_{i_{k}}\right)$. This will be viewed as analogue to $H^{2} ; \mathcal{P}$ is dense there.

Define $F^{\infty}$ as the set of all $g \in \mathcal{F}^{2}\left(\mathcal{H}_{n}\right)$ such that

$$
\|g\|_{\infty}=\sup \left\{\|g \otimes p\|_{2}: p \in \mathcal{P},\|p\|_{2} \leq 1\right\}<\infty
$$

where $\|\cdot\|_{2}=\|\cdot\|_{\mathcal{F}^{2}\left(\mathcal{H}_{n}\right)}$.

It is easy to prove (see [Po3]) that $\left(F^{\infty},\|\cdot\|_{\infty}\right)$ is a non-commutative Banach algebra (if $f, g \in F^{\infty}$, then $f \otimes g \in F^{\infty}$ and $\|f \otimes g\|_{\infty} \leq\|f\|_{\infty}\|g\|_{\infty}$ ) which can be viewed as a non-commutative analogue of the Hardy space $H^{\infty}$; when $n=1$ they coincide.

$F^{\infty}$ shares many properties with $H^{\infty}$. For instance, the second author (see [Po3]) extended the classical von Neumann's inequality [vN] to polynomials in $F^{\infty}$. He proved that if $T_{1}, \cdots, T_{n} \in B(\mathcal{H})$, for some Hilbert space $\mathcal{H}$, are such that the operator matrix $\left[T_{1} \cdots T_{n}\right]$ is a contraction (i.e., $\left\|\sum_{i=1}^{n} T_{i} T_{i}^{*}\right\| \leq 1$ ) then for every $p \in \mathcal{P}$,

$$
\left\|p\left(T_{1}, \cdots, T_{n}\right)\right\| \leq\|p\|_{\infty},
$$

where $p\left(T_{1}, \cdots, T_{n}\right)$ is obtained from (2) by replacing the $e_{i}$ 's by $T_{i}$ 's.

There are also many analogies with the invariant subspaces of $H^{2}$, inner and outer functions in $H^{\infty}$, Toeplitz operators, etc. (see [Po2], [Po4]). We will give precise definitions for many of these facts below.

For $i \leq n$ define $S_{i}: \mathcal{F}^{2}\left(\mathcal{H}_{n}\right) \rightarrow \mathcal{F}^{2}\left(\mathcal{H}_{n}\right)$ by $S_{i} \psi=e_{i} \otimes \psi$. These are unilateral shifts with orthogonal final spaces and are analogue to $S=M_{z}: H^{2} \rightarrow H^{2}$, multiplication by $z$, the unilateral shift on $H^{2}$.

For $\varphi \in F^{\infty}$ let $\varphi\left(S_{1}, \cdots, S_{n}\right): \mathcal{F}^{2}\left(\mathcal{H}_{n}\right) \rightarrow \mathcal{F}^{2}\left(\mathcal{H}_{n}\right)$ be defined by $\varphi\left(S_{1}, \cdots, S_{n}\right) \psi=$ $\varphi \otimes \psi$ for every $\psi \in \mathcal{F}^{2}\left(\mathcal{H}_{n}\right)$. Following the classical case the second author (see [Po4]) defined:

1. $\varphi \in F^{\infty}$ is inner iff $\varphi\left(S_{1}, \cdots, S_{n}\right)$ is an isometry on $\mathcal{F}^{2}\left(\mathcal{H}_{n}\right)$, and

2. $\varphi \in F^{\infty}$ is outer iff $\varphi \otimes \mathcal{F}^{2}\left(\mathcal{H}_{n}\right)$ is dense in $\mathcal{F}^{2}\left(\mathcal{H}_{n}\right)$.

In this paper we define 
In Section 1 we will set the notation, terminology and prove a few elementary facts about $\mathcal{F}^{2}\left(\mathcal{H}_{n}\right), F^{\infty}$ and inner and outer functions. In Section 2 we will study some factorization properties of inner and outer functions and will complete the characterization of the invariant subspaces for $S_{1}, \cdots, S_{n}$ started in [Po1]. In Section 3 we will provide many examples of inner functions. We will see that the theory of inner functions is more interesting in the non-commutative case than in the commutative one. In Section 4 we will prove that every weakly (strongly) closed unital subalgebra $\mathcal{A}$ of $F^{\infty}$ is reflexive; i.e., $\operatorname{Alg} \operatorname{Lat} \mathcal{A}=\mathcal{A}$. The proof of this uses most of the examples of inner functions presented in Section 3. We will finish in Section 5 with some open problems.

\section{Preliminaries}

In this section we will set the notation, terminology and prove a few elementary facts.

$\mathcal{F}^{2}\left(\mathcal{H}_{n}\right)$ is called the full Fock space on the $n$-dimensional Hilbert space $\mathcal{H}_{n}$ with orthonormal basis $\left(e_{1}, \cdots, e_{n}\right)$. It is usually represented by

$$
\mathcal{F}^{2}\left(\mathcal{H}_{n}\right)=\mathbb{C} 1 \oplus \bigoplus_{m \geq 1} \mathcal{H}_{n}^{\otimes m}
$$

Let $\Lambda=\{1,2, \cdots, n\}$ be fixed throughout the paper. For every $k \geq 1$, let $F(k, \Lambda)$ be the set of all functions from $\{1,2, \cdots, k\}$ to $\Lambda$, and let

$$
\mathcal{F}=\bigcup_{k=0}^{\infty} F(k, \Lambda),
$$

where $F(0, \Lambda)$ stands for $\{0\}$. We use $\mathcal{F}$ to describe $\mathcal{F}^{2}\left(\mathcal{H}_{n}\right)$ and simplify the notation. If $f \in F(k, \Lambda)$, let

$$
e_{f}=e_{f(1)} \otimes e_{f(2)} \otimes \cdots \otimes e_{f(k)}, \quad \text { and for } \quad k=0, \quad e_{0}=1 .
$$

Then $\mathcal{F}^{2}\left(\mathcal{H}_{n}\right)$ is the Hilbert space with orthonormal basis $\left\{e_{f}: f \in \mathcal{F}\right\}$.

Moreover, we use $\mathcal{F}$ to describe finite products. If $T_{1}, T_{2}, \cdots, T_{n} \in B(\mathcal{H})$ for some Hilbert space $\mathcal{H}$, and $f \in F(k, \Lambda)$ we denote

$$
T_{f}=T_{f(1)} T_{f(2)} \cdots T_{f(k)}, \quad T_{0}=I .
$$


Lemma 1.1. Let $\varphi, \psi \in F^{\infty}$. If $\varphi \otimes \psi=0$, then $\varphi=0$ or $\psi=0$.

Proof. Let $\varphi=\sum_{f \in \mathcal{F}} a_{f} e_{f}$ and $\psi=\sum_{g \in \mathcal{F}} b_{g} e_{g}$ be non-zero elements. Let $k$ is the smallest integer such that for some $\bar{f} \in F(k, \Lambda), a_{\bar{f}} \neq 0$, and $\ell$ the smallest integer such that for some $\bar{g} \in F(\ell, \Lambda), b_{\bar{g}} \neq 0$.

We have that $\varphi \otimes \psi=\sum_{h \in \mathcal{F}}\left[\sum_{e_{f} \otimes e_{g}=e_{h}} a_{f} b_{g}\right] e_{h}$. Let $\bar{h}$ be such that $e_{\bar{h}}=e_{\bar{f}} \otimes e_{\bar{g}}$. It is easy to see that

$$
\left\langle\varphi \otimes \psi, e_{\bar{h}}\right\rangle=\sum_{e_{f} \otimes e_{g}=e_{\bar{h}}} a_{f} b_{g}=a_{\bar{f}} b_{\bar{g}} \neq 0 .
$$

Hence, $\varphi \otimes \psi \neq 0$.

Corollary 1.2. Suppose that $\varphi \in F^{\infty}, \varphi \neq 0$ and that $\varphi=\varphi \otimes \psi$. Then $\psi=1$.

Proof. If $\varphi=\varphi \otimes \psi$, then $\varphi \otimes(1-\psi)=0$. Since $\varphi \neq 0$, we get that $1-\psi=0$.

Recall that $\varphi \in F^{\infty}$ is inner iff the map $\psi \rightarrow \varphi \otimes \psi$ is an isometry on $\mathcal{F}^{2}\left(\mathcal{H}_{n}\right)$ and that $\psi \in F^{\infty}$ is outer iff $\psi \otimes \mathcal{F}^{2}\left(\mathcal{H}_{n}\right)$ is dense in $\mathcal{F}^{2}\left(\mathcal{H}_{n}\right)$. It is immediate from the definitions that

Proposition 1.3. $\varphi$ is inner iff $\left\{\varphi \otimes e_{f}: f \in \mathcal{F}\right\}$ is an orthonormal set in $\mathcal{F}^{2}\left(\mathcal{H}_{n}\right)$.

Proposition 1.4. $\psi$ is outer iff there exist $h_{n} \in \mathcal{F}^{2}\left(\mathcal{H}_{n}\right) \quad$ (or $p_{n} \in \mathcal{P}$ ) such that $\psi \otimes h_{n} \rightarrow e_{0}\left(\psi \otimes p_{n} \rightarrow e_{0}\right)$ in $\mathcal{F}^{2}\left(\mathcal{H}_{n}\right)$.

We will also use

Proposition 1.5. Let $\varphi$ be inner. The map $\psi \rightarrow \varphi \otimes \psi$ is an isometry on $F^{\infty}$. Moreover, if $\phi=\varphi \otimes \psi$, then $\psi \in F^{\infty}$ if and only if $\phi \in F^{\infty}$ and $\|\psi\|_{\infty}=\|\phi\|_{\infty}$.

Proof. Let $\varphi$ be inner and $\psi \in F^{\infty}$. For every $p \in \mathcal{P}$ one has

$$
\|(\varphi \otimes \psi) \otimes p\|_{2}=\|\varphi \otimes(\psi \otimes p)\|_{2}=\|\psi \otimes p\|_{2}
$$

It follows from (3) that $\|\varphi \otimes \psi\|_{\infty}=\|\psi\|_{\infty}$.

Suppose now that $\phi=\varphi \otimes \psi$. It is clear that $\sup _{p \in(\mathcal{P})_{1}}\|\phi \otimes p\|_{2}$ is finite if and only if $\sup _{p \in(\mathcal{P})_{1}}\|\psi \otimes p\|_{2}$ is finite too.

Proposition 1.6. $\varphi \in F^{\infty}$ is inner iff $\|\varphi\|_{2}=\|\varphi\|_{\infty}=1$.

Proof. Suppose that $\varphi \in F^{\infty}$ is inner. Then $\|\varphi\|_{\infty} \geq\left\|\varphi \otimes e_{0}\right\|_{2}=\|\varphi\|_{2}=1$. On the other hand, if $p=\sum_{f} a_{f} e_{f} \in \mathcal{P}$, we have that $\|\varphi \otimes p\|_{2}^{2}=\sum_{f}\left|a_{f}\right|^{2}\left\|\varphi \otimes e_{f}\right\|_{2}^{2}=$ 
Conversely, suppose that $\|\varphi\|_{\infty}=\|\varphi\|_{2}=1$. Then for every $f \in \mathcal{F}, 1=$ $\|\varphi\|_{2}=\left\|\varphi \otimes e_{f}\right\|_{2}$. It is well known that if $x, y \in \ell_{2}$ satisfy $\|x\|_{2}=\|y\|_{2}=1$ and $\|x \pm y\|_{2} \leq \sqrt{2}$, then $x$ and $y$ are orthogonal. Let $f, g \in \mathcal{F}, f \neq g$. Since $\|\varphi\|_{\infty}=1$, $\left\|\varphi \otimes e_{f} \pm \varphi \otimes e_{g}\right\|_{2}=\left\|\varphi \otimes\left(e_{f} \pm e_{g}\right)\right\|_{2} \leq\left\|e_{f} \pm e_{g}\right\|_{2}=\sqrt{2}$. Therefore, $\varphi \otimes e_{f} \perp \varphi \otimes e_{g}$.

A sequence $\mathcal{S}=\left\{S_{\lambda}\right\}_{\lambda \in \Lambda}$ of unilateral shifts on a Hilbert space $\mathcal{H}$ with orthogonal final spaces is called a $\Lambda$-orthogonal shift if the operator matrix $\left[S_{1} S_{2} \cdots S_{n}\right]$ is nonunitary; i.e., $\mathcal{L}=\mathcal{H} \ominus\left(\bigoplus_{\lambda \in \Lambda} S_{\lambda} \mathcal{H}\right) \neq\{0\} . \mathcal{L}$ is called the wandering subspace of $\mathcal{S}$. The second author (see [Po2]) used $\mathcal{L}$ to get a Wold decomposition for $\mathcal{H}$. He proved that

$$
f, g \in \mathcal{F}, f \neq g \Longrightarrow S_{f} \mathcal{L} \perp S_{g} \mathcal{L}
$$

(see (4) for the notation of $S_{f}$ ), and

$$
\mathcal{H}=\bigoplus_{f \in \mathcal{F}} S_{f} \mathcal{L}
$$

Moreover the dimension of $\mathcal{L}$, called the multiplicity of the shift, determines up to unitary equivalence the $\Lambda$-orthogonal shift.

A model for the $\Lambda$-orthogonal shifts of multiplicity one is provided by the unilateral shifts $S_{i}: \mathcal{F}^{2}\left(\mathcal{H}_{n}\right) \rightarrow \mathcal{F}^{2}\left(\mathcal{H}_{n}\right)$ defined by $S_{i} \psi=e_{i} \otimes \psi, i \leq n$. These operators are also called the left creation operators on the full Fock space.

Let $U: \mathcal{F}^{2}\left(\mathcal{H}_{n}\right) \rightarrow \mathcal{F}^{2}\left(\mathcal{H}_{n}\right)$ be the flip operator defined by $U \varphi=\tilde{\varphi}$ where

$$
e_{f}=e_{f(1)} \otimes \cdots \otimes e_{f(k)}, \quad U e_{f}=\tilde{e}_{f}=e_{f(k)} \otimes e_{f(k-1)} \otimes \cdots \otimes e_{f(1)} .
$$

It is easy to check that $U$ is unitary and that $U=U^{*}=U^{-1}$. We use $U$ to describe right multiplication on $\mathcal{F}^{2}\left(\mathcal{H}_{n}\right)$.

Lemma 1.7. Let $\psi \in \mathcal{F}^{2}\left(\mathcal{H}_{n}\right)$ and suppose that

$$
\sup \left\{\|p \otimes \psi\|_{2}: p \in \mathcal{P},\|p\|_{2} \leq 1\right\}<\infty .
$$

Then $\tilde{\psi} \in F^{\infty}$ and the supremum is equal to $\|\tilde{\psi}\|_{\infty}$.

Proof. Since $U$ is an isometry on $\mathcal{F}^{2}\left(\mathcal{H}_{n}\right)$ and $U(p \otimes q)=U(q) \otimes U(p)=\tilde{q} \otimes \tilde{p}$, we have

$$
\sup \|p \otimes \psi\|_{2}=\sup \|\tilde{\psi} \otimes \tilde{p}\|_{2}=\sup \|\tilde{\psi} \otimes p\|_{2}=\|\tilde{\psi}\|_{\infty} .
$$


Remark. $U$ is unbounded in $F^{\infty}$. Example 6 of Section 3 tells us that if $p\left(e_{1}\right)$ is a polynomial in $e_{1}$, then $\left\|e_{2} \otimes p\left(e_{1}\right)\right\|_{\infty}=\left\|p\left(e_{1}\right)\right\|_{\infty}$ but $\left\|U\left(e_{2} \otimes p\left(e_{1}\right)\right)\right\|_{\infty}=$ $\left\|p\left(e_{1}\right) \otimes e_{2}\right\|_{\infty}=\left\|p\left(e_{1}\right)\right\|_{2}$.

\section{FACTORIZATion RESUlts}

In this section we will prove

Theorem 2.1. If $\psi \in F^{2}\left(H_{n}\right), \psi \neq 0$, then there exist $\varphi$ inner and $g$ outer functions such that $\psi=\varphi \otimes g$. Moreover, the factorization is essentially unique and $\psi \in F^{\infty}$ if and only if $g \in F^{\infty}$ and $\|\psi\|_{\infty}=\|g\|_{\infty}$.

And together with [Po3, Corollary 3.5],

Theorem 2.2. Let $\Phi: F^{\infty} \rightarrow B\left(\mathcal{F}^{2}\left(H_{n}\right)\right)$ be defined by $\Phi(\varphi)=\varphi\left(S_{1}, \cdots, S_{n}\right)$. Then:

(i) $\Phi$ is an algebra homomorphism.

(ii) $\|\Phi \psi\|=\|\psi\|_{\infty}$, for any $\psi \in F^{\infty}$.

(iii) $\psi$ is invertible in $F^{\infty}$ if and only if $\Phi \psi$ is invertible in $B\left(\mathcal{F}^{2}\left(H_{n}\right)\right)$.

To prove Theorem 2.1 we will first complete the characterization of invariant subspaces for $S_{1}, \cdots, S_{n}$ started in [Po1] for $\Lambda$-orthogonal shifts of arbitrary multiplicity.

We say that the inner functions $\varphi_{1}, \varphi_{2}$ are orthogonal if $\varphi_{1} \otimes \mathcal{F}^{2}\left(\mathcal{H}_{n}\right) \perp \varphi_{2} \otimes$ $\mathcal{F}^{2}\left(\mathcal{H}_{n}\right)$.

Theorem 2.3. If $\mathcal{M} \subset \mathcal{F}^{2}\left(H_{n}\right)$ is invariant for each $S_{1}, \cdots, S_{n}$ then there exists a sequence $\{\varphi\}_{j \in J}$ of orthogonal inner functions such that

$$
\mathcal{M}=\bigoplus_{j \in J} F^{2}\left(H_{n}\right) \otimes \tilde{\varphi}_{j}
$$

Moreover, this representation is essentially unique.

Proof. Let $\mathcal{M}$ be a nontrivial closed invariant subspace for $S_{1}, \cdots, S_{n}$. According to $(6)$

$$
\mathcal{M}=\bigoplus_{f \in \mathcal{F}} S_{f} \mathcal{L}
$$


Let $\left\{\tilde{\varphi}_{j}\right\}_{j \in J}$ be an orthonormal basis in $\mathcal{L}$. According to (5), for each $j \in J$

$$
S_{f} \tilde{\varphi}_{j} \perp S_{g} \tilde{\varphi}_{j}, \quad \text { for any } \quad f, g \in \mathcal{F}, f \neq g .
$$

By Proposition 1.3 we infer that $\varphi_{j}$ is an inner function. On the other hand the relations (7) and (8) imply the following orthogonal decomposition

$$
\mathcal{M}=\bigoplus_{j \in J} F^{2}\left(H_{n}\right) \otimes \tilde{\varphi}_{j}
$$

Suppose that $\mathcal{M}=\bigoplus_{i \in I}\left[\mathcal{F}^{2}\left(\mathcal{H}_{n}\right) \otimes \tilde{\psi}_{i}\right]$ for some orthogonal inner functions $\psi_{i}$. Let $\mathcal{L}^{\prime}=\vee_{i \in I} \tilde{\psi}_{i}$. It is easy to see that

$$
\mathcal{M}=\bigoplus_{f \in S_{f}} S_{f} \mathcal{L}^{\prime}
$$

This implies that $\mathcal{L}^{\prime}=\mathcal{M} \ominus\left(S_{1} \mathcal{M} \oplus \cdots \oplus S_{n} \mathcal{M}\right)$ is the wandering subspace for $\left.S_{1}\right|_{\mathcal{M}}, \cdots,\left.S_{n}\right|_{\mathcal{M}}$ and $\mathcal{L}=\mathcal{L}^{\prime}$. Since $\left\{\tilde{\varphi}_{j}\right\}_{j \in J}$ and $\left\{\tilde{\psi}_{i}\right\}_{i \in I}$ are orthogonormal basis in $\mathcal{L}$ we infer that they have the same cardinality and that there exists a unitary operator $U \in B(\mathcal{L})$ such that $U \tilde{\varphi}_{j}=\tilde{\psi}_{j}, j \in J$.

Corollary 2.4. If $\varphi_{1}, \varphi_{2}$ are inner functions such that $\varphi_{1} \otimes \mathcal{F}^{2}\left(\mathcal{H}_{n}\right)=\varphi_{2} \otimes$ $\mathcal{F}^{2}\left(\mathcal{H}_{n}\right)$, then there exists $\alpha \in \mathbb{C},|\alpha|=1$ such that $\varphi_{1}=\alpha \varphi_{2}$.

Proof. Let $\mathcal{M}=\mathcal{F}^{2}\left(\mathcal{H}_{n}\right) \otimes \tilde{\varphi}_{1}=\mathcal{F}^{2}\left(\mathcal{H}_{n}\right) \otimes \tilde{\varphi}_{2}$. It is clear that $\mathcal{M}$ is invariant for $S_{1}, \cdots, S_{n}$. It follows from the proof of Theorem 2.3 that $\left[\tilde{\varphi}_{1}\right]=\left[\tilde{\varphi}_{2}\right]$. Since $\tilde{\varphi}_{1}, \tilde{\varphi}_{2}$ are normalized in $\mathcal{F}^{2}\left(\mathcal{H}_{n}\right)$ there exists $\alpha \in \mathbb{C},|\alpha|=1$ such that $\tilde{\varphi}_{1}=\alpha \tilde{\varphi}_{2}$.

The next lemma will show that all the cyclic invariant subspaces of $\mathcal{F}^{2}\left(\mathcal{H}_{n}\right)$ are of the form $\mathcal{F}^{2}\left(\mathcal{H}_{n}\right) \otimes \tilde{\varphi}$ for some inner function $\varphi$.

Lemma 2.5. Let $\mathcal{V}=\left\{V_{1}, \cdots, V_{n}\right\}$ be a $\Lambda$-orthogonal shift of arbitrary multiplicity acting on the Hilbert space $\mathcal{K}$. If $\mathcal{M} \subset \mathcal{K}$ is a cyclic invariant subspace of $\mathcal{V}$, that is

$$
\mathcal{M}=\bigvee_{f \in \mathcal{F}} V_{f} \psi, \text { for some } \psi \in \mathcal{K}, \psi \neq 0
$$

then $\mathcal{V}_{\mid \mathcal{M}}:=\left\{\left.V_{1}\right|_{\mathcal{M}}, \cdots,\left.V_{n}\right|_{\mathcal{M}}\right\}$ is unitarily equivalent to the $\Lambda$-orthogonal shift of multiplicity 1 .

Proof. Let $\psi \in \mathcal{K}, \psi \neq 0$ be a fixed element in $\mathcal{K}$ and let

$$
\mathcal{M}=\bigvee V_{f} \psi
$$


Since $\mathcal{V}$ is a $\Lambda$-orthogonal shift in $\mathcal{K}$ it follows from (5) and (6) that $\mathcal{K}=$ $\bigoplus_{f \in \mathcal{F}} V_{f} \mathcal{L}$, where $\mathcal{L}=\mathcal{K} \ominus\left(V_{1} \mathcal{K} \oplus \cdots \oplus \mathcal{V}_{k} \mathcal{K}\right)$ is the wandering subspace of $\mathcal{V}$.

Since $\left.\mathcal{V}\right|_{\mathcal{M}}$ is also a $\Lambda$-orthogonal shift let us denote by $\mathcal{L}_{0}$ its wandering subspace. Therefore, $\mathcal{L}_{0} \subset \mathcal{M}$ and

$$
\mathcal{M}=\bigoplus_{f \in \mathcal{F}} V_{f} \mathcal{L}_{0}, \quad \text { where } \quad \mathcal{L}_{0}=\mathcal{M} \ominus\left(V_{1} \mathcal{M} \oplus \cdots \oplus V_{n} \mathcal{M}\right) .
$$

Consider $w_{0}=P_{\mathcal{L}_{0}} \psi$, where $P_{\mathcal{L}_{0}}$ is the orthogonal projection from $\mathcal{K}$ onto $\mathcal{L}_{0}$. Notice that according to $(9)$ it follows that $w_{0} \neq 0$. Let $\ell_{0} \in \mathcal{L}_{0}$ be such that $\ell_{0} \perp w_{0}$. Since $\mathcal{L}_{0}$ is wandering subspace for $\left.\mathcal{V}\right|_{\mathcal{M}}$ we have

$$
\ell_{0} \perp V_{f} \psi, \quad \text { for any } f \in F(k, \Lambda), k \geq 1 \text {. }
$$

On the other hand

$$
\left\langle\ell_{0}, \psi\right\rangle=\left\langle\ell_{0}, P_{\mathcal{L}_{0}} \psi\right\rangle=\left\langle\ell_{0}, w_{0}\right\rangle=0 .
$$

Therefore, $\quad \ell_{0} \perp V_{f} \psi$, for any $f \in \mathcal{F}$. Since $\ell_{0} \in \mathcal{M}=\bigvee_{f \in \mathcal{F}} V_{f} \psi \quad$ we infer that $\ell_{0}=0$. Thus, $\operatorname{dim} \mathcal{L}_{0}=1$ and according to [Po3, Theorem 1.2] it follows that $\left.\mathcal{V}\right|_{\mathcal{M}}$ is unitarily equivalent to the $\Lambda$-orthogonal shift of multiplicity one $\mathcal{S}=\left\{S_{1}, \cdots, S_{n}\right\}$. The proof is complete.

Corollary 2.6. If $\psi \in F^{2}\left(H_{n}\right), \psi \neq 0$ then there exists an inner function $\varphi \in F^{\infty}$ such that

$$
\operatorname{clos}[\mathcal{P} \otimes \psi]=F^{2}\left(H_{n}\right) \otimes \tilde{\varphi} .
$$

Moreover, the representation is essentially unique.

Proof. Let $\mathcal{M}=\operatorname{clos}[\mathcal{P} \otimes \psi]$. It follows from the previous lemma that $\mathcal{L}=\mathcal{M} \ominus$ $\left(S_{1} \mathcal{M} \oplus \cdots \oplus S_{n} \mathcal{M}\right)$, the wandering subspace for $\left.S_{1}\right|_{\mathcal{M}}, \cdots,\left.S_{n}\right|_{\mathcal{M}}$, has dimension 1. Using Proposition 2.3 we get that $\mathcal{M}=\mathcal{F}^{2}\left(\mathcal{H}_{n}\right) \otimes \tilde{\varphi}$ for some inner function $\varphi$. The "uniqueness" is a consequence of Corollary 2.4.

Proof of Theorem 2.1. Let $\mathcal{M}=\operatorname{clos}[\mathcal{P} \otimes \tilde{\psi}]$. Then $\mathcal{M}$ is a nontrivial closed invariant subspace for each $S_{1}, \cdots, S_{n}$. By Corollary 2.6 it is of the form $\mathcal{M}=F^{2}\left(H_{n}\right) \otimes \tilde{\varphi}$, where $\varphi \in F^{\infty}$ is inner. Since $\tilde{\psi} \in \mathcal{M}$, there must exist $\tilde{g} \in F^{2}\left(H_{n}\right)$ such that 
Since $\tilde{\varphi} \in \mathcal{M}$, there exist $p_{n} \in \mathcal{P}$ such that

$$
\tilde{\varphi}=\lim _{n \rightarrow \infty} p_{n} \otimes \tilde{\psi}=\lim _{n \rightarrow \infty}\left(p_{n} \otimes \tilde{g}\right) \otimes \tilde{\varphi}=\left[\lim _{n \rightarrow \infty} p_{n} \otimes \tilde{g}\right] \otimes \tilde{\varphi} .
$$

We use that $\varphi$ is inner for the last equality. By Corollary $1.2, \lim _{n \rightarrow \infty} p_{n} \otimes \tilde{g}=e_{0}$. Hence $\lim _{n \rightarrow \infty} g \otimes \tilde{p}_{n}=e_{0}$, and using Proposition 1.4 we conclude that $g$ is outer. It follows from Proposition 1.5 that $g \in F^{\infty}$ iff $\psi \in F^{\infty}$.

Suppose now that $\psi=\varphi_{1} \otimes g_{1}=\varphi_{2} \otimes g_{2}$ for some $\varphi_{1}, \varphi_{2}$ inner and $g_{1}, g_{2}$ outer. Then we have that $\varphi_{1} \otimes \mathcal{F}^{2}\left(\mathcal{H}_{n}\right)=\varphi_{2} \otimes \mathcal{F}^{2}\left(\mathcal{H}_{n}\right)$, and using Corollary 2.4 we get that $\varphi_{1}=\alpha \varphi_{2}$ for some $\alpha \in \mathbb{C},|\alpha|=1$. Hence $0=\varphi_{1} \otimes g_{1}-\varphi_{2} \otimes g_{2}=\varphi_{2} \otimes\left(\alpha g_{1}-g_{2}\right)$. By Lemma 1.1, $\alpha g_{1}=g_{2}$.

As in the classical case, there is a factorization result for inner functions.

Corollary 2.7. Let $\varphi_{1}, \varphi_{2}$ be inner functions. $\varphi_{1} \otimes \mathcal{F}^{2}\left(\mathcal{H}_{n}\right) \subset \varphi_{2} \otimes \mathcal{F}^{2}\left(\mathcal{H}_{n}\right)$ if and only if there exists $\varphi_{3}$ inner such that $\varphi_{1}=\varphi_{2} \otimes \varphi_{3}$.

Proof. Suppose that $\varphi_{1} \otimes \mathcal{F}^{2}\left(\mathcal{H}_{n}\right) \subset \varphi_{2} \otimes \mathcal{F}^{2}\left(\mathcal{H}_{n}\right)$. Then $\varphi_{1}=\varphi_{2} \otimes \psi$ for some $\psi \in \mathcal{F}^{2}\left(\mathcal{H}_{n}\right)$. Let $\psi=\psi_{i} \otimes \psi_{e}$ be the inner-outer factorization of $\psi$. Therefore, $\varphi_{1}=\left(\varphi_{2} \otimes \psi_{i}\right) \otimes \psi_{e}$. By the uniqueness of the factorization of $\varphi_{1}$ we deduce that $\psi_{e}=1$. The converse is clear.

We will finish the details of Theorem 2.2 now. Parts (i) and (ii) appear in [Po3]. The third part is included in the proof of the next theorem.

Theorem 2.8. $\varphi \in F^{\infty}$ is invertible if and only if $\varphi$ is outer and there exists $\delta>0$ such that $\|\varphi \otimes p\|_{2} \geq \delta\|p\|_{2}$, for any $p \in \mathcal{P}$.

Proof.. Let $\varphi \in F^{\infty}$ be invertible and find $\psi \in F^{\infty}$ such that $\varphi \otimes \psi=\psi \otimes \varphi=e_{0}$. Hence we infer that

$$
\varphi\left(S_{1}, \cdots, S_{n}\right) \psi\left(S_{1}, \cdots, S_{n}\right)=\psi\left(S_{1}, \cdots, S_{n}\right) \varphi\left(S_{1}, \cdots, S_{n}\right)=I_{F^{2}\left(H_{n}\right)} .
$$

Therefore the operator $\varphi\left(S_{1}, \cdots, S_{n}\right)$ is invertible which implies:

$$
\text { range } \varphi\left(S_{1}, \cdots, S_{n}\right)=F^{2}\left(H_{n}\right), \quad \text { and }
$$


The relation (10) shows that $\varphi$ is outer and (11) implies that there exists $\delta>0$ such that

$$
\|\varphi \otimes p\|_{2} \geq \delta\|p\|_{2}, \quad \text { for any } p \in \mathcal{P}
$$

Conversely, assume $\varphi \in F^{\infty}$ is outer such that (12) holds. This implies that $\varphi\left(S_{1}, \cdots, S_{n}\right)$ is invertible in $B\left(F^{2}\left(H_{n}\right)\right)$. Let $T \in B\left(F^{2}\left(H_{n}\right)\right)$ be such that

$$
T \varphi\left(S_{1}, \cdots, S_{n}\right)=\varphi\left(S_{1}, \cdots, S_{n}\right) T=I_{F^{2}\left(H_{n}\right)}
$$

Then for any $h \in F^{2}\left(H_{n}\right)$ we have

$$
T(\varphi \otimes h)=\varphi \otimes T h=h
$$

Let $h=e_{0}$ and $\psi=T e_{0}$. Then (13) gives us

$$
\varphi \otimes \psi=e_{0} \quad \text { and } \quad T(\varphi)=e_{0}
$$

We clearly have

$$
\varphi=e_{0} \otimes \varphi=(\varphi \otimes \psi) \otimes \varphi=\varphi \otimes(\psi \otimes \varphi)
$$

Then according to (14) and (13) we have $e_{0}=T(\varphi)=T(\varphi \otimes(\psi \otimes \varphi))=\psi \otimes \varphi$. Therefore,

$$
\varphi \otimes \psi=\psi \otimes \varphi=e_{0}
$$

We claim that $\psi \in F^{\infty}$. Let $p \in \mathcal{P}$. It is clear that

$$
p=e_{0} \otimes p=(\varphi \otimes \psi) \otimes p=\varphi \otimes(\psi \otimes p)
$$

Therefore, by (13)

$$
T(p)=T(\varphi \otimes(\psi \otimes p))=\psi \otimes p
$$

Hence,

$$
\|\psi\|_{\infty}=\sup _{p \in(\mathcal{P})_{1}}\|\psi \otimes p\|_{2}=\sup _{p \in(\mathcal{P})_{1}}\|T p\|_{2}=\|T\|<\infty .
$$




\section{EXAMPLES OF INNER AND OUTER FUNCTIONS}

In this section we will present many examples of inner and outer functions in $F^{\infty}$. Recall that $\varphi \in F^{\infty}$ is inner iff the map $h \rightarrow \varphi \otimes h$ is an isometry on $\mathcal{F}^{2}\left(\mathcal{H}_{n}\right)$, (equivalently, $h \rightarrow h \otimes \tilde{\varphi}$ is an isometry on $\mathcal{F}^{2}\left(\mathcal{H}_{n}\right)$ ), and $\varphi \in F^{\infty}$ is outer iff there exists $p_{n} \in \mathcal{F}^{2}\left(\mathcal{H}_{n}\right)$ such that $\varphi \otimes p_{n} \rightarrow e_{0}$ in $\mathcal{F}^{2}\left(H_{n}\right)$.

The first observation is that any inner (respectively outer) function in $H^{\infty}$ becomes inner (respectively outer) in $F^{\infty}$ if we substitute $z$ by $e_{f}$. We call these examples "inherited".

Example 1. Inherited inner and outer functions.

For any $f \in \mathcal{F}, f \neq 0$ let $V_{f}: H^{2} \rightarrow \mathcal{F}^{2}\left(H_{n}\right)$ be the isometry defined by $V_{f} z^{k}=e_{f}^{k}$. We denote $V_{f} \varphi=\varphi_{f}$.

Proposition 3.1. $V_{f}: H^{\infty} \rightarrow F^{\infty}$ is an isometry. Moreover, if $\varphi \in H^{\infty}$ is inner then $\varphi_{f}$ is inner in $F^{\infty}$, and if $\varphi \in H^{\infty}$ is outer then $\varphi_{f}$ is outer in $F^{\infty}$.

Proof. Let $\mathcal{F}_{f}^{2}=\left[e_{0}, e_{f}, e_{f}^{2}, \cdots\right]$. Any $h \in \mathcal{F}$ satisfies $e_{h}=e_{f}^{k} \otimes e_{g}$ for some $k \in \mathbb{N}_{0}$ and $g \in \mathcal{F}$ that "does-not-start" from $f$ (i.e., $S_{f}^{*} e_{g}=0$, see (4) for the notation of $\left.S_{f}\right)$. We decompose $\mathcal{F}^{2}\left(\mathcal{H}_{n}\right)$ as

$$
\mathcal{F}^{2}\left(\mathcal{H}_{n}\right)=\bigoplus_{\substack{g \in \mathcal{F} \\ S_{f}^{*} e_{g}=0}}\left[\mathcal{F}_{f}^{2} \oplus e_{g}\right]
$$

Since $\varphi_{f} \in \mathcal{F}_{f}^{2}$ we have that for any $g \in \mathcal{F}$ that does not start from $f$,

$$
\varphi_{f} \otimes\left[\mathcal{F}_{f}^{2} \otimes e_{g}\right] \subset \mathcal{F}_{f}^{2} \otimes e_{g}
$$

Moreover, it is easy to see that

$$
\sup _{p \in\left(\mathcal{F}_{f}^{2} \otimes e_{g}\right)_{1}}\left\|\varphi_{f} \otimes p\right\|_{2}=\sup _{p \in\left(\mathcal{F}_{f}^{2}\right)_{1}}\left\|\varphi_{f} \otimes p\right\|_{2}=\sup _{p \in\left(H^{2}\right)_{1}}\|\varphi p\|_{2}=\|\varphi\|_{\infty}
$$

Combining this with the fact that $\varphi_{f}$ acts diagonally in the decomposition of $\mathcal{F}^{2}\left(\mathcal{H}_{n}\right)$ given by (15) we conclude that $\left\|\varphi_{f}\right\|_{\infty}=\|\varphi\|_{\infty}$.

Suppose that $\varphi \in H^{\infty}$ is outer. Then we can find a sequence of $p_{n} \in H^{2}$ such that $\varphi p_{n} \rightarrow 1$ in $H^{2}$. Since $V_{f}$ is an isometry on $H^{2}$ we get that $V_{f}\left(\varphi p_{n}\right)=$ 
Suppose now that $\varphi \in H^{\infty}$ is inner. We want to prove that whenever $h_{1}, h_{2} \in \mathcal{F}$ satisfy $h_{1} \neq h_{2}$, we will have that $\varphi_{f} \otimes e_{h_{1}} \perp \varphi_{f} \otimes e_{h_{2}}$.

Consider first the case $e_{h_{1}}=e_{f}^{k}, e_{h_{2}}=e_{f}^{\ell}$ for $k \neq \ell$. Since $\varphi \in H^{\infty}$ is inner then $\varphi z^{k} \perp \varphi z^{\ell}$. Hence using that $V_{f}$ is an isometry we get that $\varphi_{f} \otimes e_{h_{1}} \perp \varphi_{f} \otimes e_{h_{2}}$.

Suppose now that $e_{h_{i}} \in \mathcal{F}_{f}^{2} \otimes e_{g_{i}}, i=1,2$ for some $g_{1}, g_{2} \in \mathcal{F}$ that do not start from $f$.

If $g_{1} \neq g_{2}$ then $\varphi_{f} \otimes e_{h_{i}} \in \mathcal{F}_{f}^{2} \otimes e_{g_{i}}$ for $i=1,2$. Since these two subspaces are orthogonal we conclude that $\varphi_{f} \otimes e_{h_{1}} \perp \varphi_{f} \otimes e_{h_{2}}$.

If $g_{1}=g_{2}$ then $e_{h_{1}}=e_{f}^{k} \otimes e_{g}$ and $e_{h_{2}}=e_{f}^{\ell} \otimes e_{g}$ for some $k \neq \ell$. Hence,

$$
\left\langle\varphi_{f} \otimes e_{h_{1}}, \varphi_{f} \otimes e_{h_{2}}\right\rangle=\left\langle\varphi_{f} \otimes e_{f}^{k}, \varphi_{f} \otimes e_{f}^{\ell}\right\rangle=0
$$

The last equality follows from the first case.

Example 2. (Invertible elements) If $\psi \in F^{\infty}$ is invertible, then $\psi$ is outer.

In particular, the following examples are invertible (and hence outer):

(i) For $\varphi_{i} \in F^{\infty},\left\|\varphi_{i}\right\|_{\infty}<1$ for $i \leq k$, let

$$
\psi=\left(e_{0}-\varphi_{1}\right) \otimes\left(e_{0}-\varphi_{2}\right) \otimes \cdots \otimes\left(e_{0}-\varphi_{k}\right) .
$$

(ii) For $\varphi \in F^{\infty}$, and $\varphi^{n}=\varphi \otimes \cdots \otimes \varphi(n$-times), let

$$
\psi=\exp \varphi=\sum_{k=0}^{\infty} \frac{\varphi^{n}}{n !} .
$$

(iii) For $\lambda \in \mathbb{C}^{n},\|\lambda\|_{2}<1$ let $\psi=z_{\lambda}$, where the $z_{\lambda}$ 's are defined in Example 8 .

We will present examples of inner functions now. The first example is the simplest.

Example 3. (The monomials) For every $f \in \mathcal{F}, e_{f}$ is inner.

Examples 4 and 6 below appear in $[\mathrm{A}]$.

Example 4. For every $k \in \mathbb{N}$ let $X_{k}=\operatorname{span}\left\{e_{f}: f \in F(k, \Lambda)\right\}$ (i.e., the span of the monomials with $k$ letters). Then any $x \in X_{k},\|x\|_{2}=1$ is inner.

Proof. The main point is that if $f_{1}, f_{2} \in F(k, \Lambda)$ and $g, h \in \mathcal{F}$ then $e_{f_{1}} \otimes e_{g}=$ $e_{f_{2}} \otimes e_{h}$ iff $f_{1}=f_{2}$ and $g=h$.

We can easily see that if $x=\sum_{f \in F(k, \Lambda)} a_{f} e_{f} \in X_{k}$ and $g, h \in \mathcal{F}, g \neq h$ then $x \otimes e_{g}$ is orthogonal to $x \otimes e_{h}$. If we require that $\|x\|_{2}=1, x$ is inner. 
Example 5. Let $e_{f_{1}}, \cdots, e_{f_{k}}$ be monomials such that the first letter of all of them are different. (i.e., $k \neq \ell$ implies that $f_{k}(1) \neq f_{\ell}(1)$ ). Then for any $\sum_{i \leq k}\left|a_{i}\right|^{2}=1$ we have that $\sum_{i \leq k} a_{i} e_{f_{i}}$ is inner.

Proof. The proof is similar to that of Example 4. Since the $f_{i}$ 's start from different letters, for any $g, h \in \mathcal{F}, e_{f_{i}} \otimes e_{g} \perp e_{f_{j}} \otimes e_{h}$ if $i \neq j$.

We can easily see that if $x=\sum_{i \leq k} a_{i} e_{f_{i}}$ and $g, h \in \mathcal{F}, g \neq h$ then $x \otimes e_{g}$ is orthogonal to $x \otimes e_{h}$. If we require that $\|x\|_{2}=1, x$ is inner.

Example 6. Let $\psi \in \mathcal{F}^{2}\left(H_{n-1}\right),\|\psi\|_{2}=1$, (i.e., $\psi$ does not have any $\left.e_{n}\right)$. Then $\psi \otimes e_{n}$ is inner.

Proof. Let $\mathcal{F}_{n-1}$ be the set of words on the letters $e_{1}, \cdots, e_{n-1}$. Let $f_{1}, f_{2} \in \mathcal{F}_{n-1}$ and $g_{1}, g_{2} \in \mathcal{F}$. The main point is that

$$
e_{f_{1}} \otimes e_{n} \otimes e_{g_{1}}=e_{f_{2}} \otimes e_{n} \otimes e_{g_{2}} \quad \text { if and only if } \quad f_{1}=f_{2} \text { and } g_{1}=g_{2} \text {. }
$$

To see this notice that the first time that $e_{n}$ appears in $e_{f_{1}} \otimes e_{n} \otimes e_{g_{1}}$ is right after $e_{f_{1}}$. Since the same is true for $f_{2}$ the words must agree before $e_{n}$ (i.e., $f_{1}=f_{2}$ ) and after (i.e., $\left.g_{1}=g_{2}\right)$.

It is easy to prove now that if $g_{1}, g_{2} \in \mathcal{F}, g_{1} \neq g_{2}$, then $\psi \otimes e_{n} \otimes e_{g_{1}} \perp \psi \otimes e_{n} \otimes e_{g_{2}}$. The normalization condition guarantees that $\psi \otimes e_{n}$ is inner.

Example 7. (Möbius functions) For any $f \in \mathcal{F}$, and $\mu \in \mathbb{C}$ with $|\mu|<1$, we have that $\varphi(f, \mu)=\left(e_{f}-\mu\right) \otimes\left(1-\bar{\mu} e_{f}\right)^{-1}$ is inner.

These are particular cases for Example 1 above. The products of these elements can be viewed as analogue to the Blashke products. However, $\left[\varphi(f, \mu) \otimes \mathcal{F}^{2}\left(\mathcal{H}_{n}\right)\right]^{\perp}$ is always infinite codimensional. In fact, since $\left(1-\bar{\mu} e_{f}\right)^{-1}$ is invertible and $\left(1-\bar{\mu} e_{f}\right)^{-1}$, $\left(e_{f}-\mu\right)$ commute, we have that $h \in\left[\varphi(f, \mu) \otimes \mathcal{F}^{2}\left(\mathcal{H}_{n}\right)\right]^{\perp}$ iff for every $\psi \in \mathcal{F}^{2}\left(\mathcal{H}_{n}\right)$, $\left\langle\left(e_{f}-\mu\right) \otimes \psi, h\right\rangle=0$. Then, looking only at the basic elements we get that

$$
h \in\left[\varphi(f, \mu) \otimes \mathcal{F}^{2}\left(\mathcal{H}_{n}\right)\right]^{\perp} \Longleftrightarrow \forall g \in \mathcal{F},\left\langle e_{f} \otimes e_{g}, h\right\rangle=\mu\left\langle e_{g}, h\right\rangle .
$$

It is easy to see that

$$
h_{f}(\mu)=\sum_{k=0}^{\infty} \mu^{k} e_{f}^{k}
$$


One can also check that if $g \in \mathcal{F}$ "does-not-start" from $f$ (i.e., $S_{f}^{*} e_{g}=0$, see (4) for the notation of $\left.S_{f}\right)$, then $h_{f}(\mu) \otimes e_{g} \in\left[\varphi(f, \mu) \otimes \mathcal{F}^{2}\left(\mathcal{H}_{n}\right)\right]^{\perp}$. Moreover, the span of these elements is dense in $\left[\varphi(f, \mu) \otimes \mathcal{F}^{2}\left(\mathcal{H}_{n}\right)\right]^{\perp}$. We leave the details out.

We need the following lemma in the next section.

Lemma 3.2. Let $\Omega \subset\{\mu \in \mathbb{C}:|\mu|<1\}$ be a set with an accumulation point in $\{\mu \in \mathbb{C}:|\mu|<1\}$. Then $\bigcap\left\{\varphi(f, \mu) \otimes \mathcal{F}^{2}\left(\mathcal{H}_{n}\right): f \in \mathcal{F}, \mu \in \Omega\right\}=\{0\}$.

Proof. Let $\psi \in \bigcap\left\{\varphi(f, \mu) \otimes \mathcal{F}^{2}\left(\mathcal{H}_{n}\right): f \in \mathcal{F}, \mu \in \Omega\right\}$ and fix $f \in \mathcal{F}$. For every $\mu \in \Omega$, we have that $\left\langle\psi, h_{f}(\mu)\right\rangle=0$. Hence,

$$
\left\langle\psi, e_{0}\right\rangle+\mu\left\langle\psi, e_{f}\right\rangle+\mu^{2}\left\langle\psi, e_{f}^{2}\right\rangle+\mu^{3}\left\langle\psi, e_{f}^{3}\right\rangle+\cdots=0 \quad \text { for all } \quad \mu \in \Omega
$$

Since the map $\mu \rightarrow \sum_{k=0}^{\infty} \mu^{k}\left\langle\psi, e_{f}^{k}\right\rangle$ is analytic on $\mu$ and the zeros accumulate inside the disk, the map is zero. Hence $\left\langle\psi, e_{0}\right\rangle=\left\langle\psi, e_{f}\right\rangle=0$. Since $f$ is arbitrary, we conclude that $\psi=0$.

The following example is more technical and it is not used in the rest of the paper. However, it gives rise to non-trivial inner functions and to an interesting ideal in $F^{\infty}$ that seems to capture the "non-commutativity" of the product.

Example 8. 1-codimensional invariant subspaces.

Let $\mathcal{M}$ be a 1-codimensional subpace of $\mathcal{F}^{2}\left(\mathcal{H}_{n}\right)$ invariant for $S_{1}, \cdots, S_{n}$. Then $\mathcal{M}=[z]^{\perp}$ for some $z \in \mathcal{F}^{2}\left(\mathcal{H}_{n}\right)$ and $[z]$ is invariant for $S_{1}^{*}, \cdots, S_{n}^{*}$. That is, for every $i \leq n$ there exists $\lambda_{i} \in \mathbb{C}$ such that

$$
S_{i}^{*} z=\lambda_{i} z
$$

Assume that $\left\langle z, e_{0}\right\rangle=1$.

For $f \in F(k, \Lambda)$ let $\lambda_{f}=\lambda_{f(1)} \lambda_{f(2)} \cdots \lambda_{f(k)}$ and $\lambda_{0}=1$. We claim that for any $f \in \mathcal{F}$

$$
\left\langle z, e_{f}\right\rangle=\lambda_{f} \quad \text { and then } \quad z=\sum_{f \in \mathcal{F}} \lambda_{f} e_{f}
$$

To see this notice that for every $i \leq n,\left\langle z, e_{i}\right\rangle=\left\langle z, S_{i} e_{0}\right\rangle=\left\langle S_{i}^{*} z, e_{0}\right\rangle=\lambda_{i}$. Similarly, $\left\langle z, e_{i} \otimes e_{j}\right\rangle=\left\langle z, S_{i} S_{j} e_{0}\right\rangle=\left\langle S_{j}^{*} S_{i}^{*} z, e_{0}\right\rangle=\lambda_{i} \lambda_{j}$. Proceeding inductively we get (17). Since the $\lambda_{i}$ 's determine $z$ and $\mathcal{M}$ we will denote them by $z_{\lambda}$ and $\mathcal{M}_{\lambda}$ from now 
It is not hard to see that

$$
z_{\lambda}=\sum_{f \in \mathcal{F}} \lambda_{f} e_{f}=1+\sum_{k=1}^{\infty}\left(\lambda_{1} e_{1}+\lambda_{2} e_{2} \cdots+\lambda_{n} e_{n}\right)^{k}
$$

Then a necessary and sufficient condition for $z_{\lambda} \in \mathcal{F}^{2}\left(\mathcal{H}_{n}\right)$ is that $\|\lambda\|_{2}=\sqrt{\sum_{i \leq n}\left|\lambda_{i}\right|^{2}}<$ 1. Moreover, by Example 4 and Proposition 1.6, $\left\|\lambda_{1} e_{1}+\cdots \lambda_{n} e_{n}\right\|_{2}=\| \lambda_{1} e_{1}+$ $\cdots \lambda_{n} e_{n} \|_{\infty}$ and then $z_{\lambda} \in F^{\infty}$.

We have thus proved:

Proposition 3.3. $\mathcal{M}$ is a 1-codimensional invariant subspace for $S_{1}, \cdots, S_{n}$ if and only if $\mathcal{M}=\left[z_{\lambda}\right]^{\perp}$ for some $\lambda \in \mathbb{C}^{n},\|\lambda\|_{2}<1$.

It follows from Proposition 2.3 that $\mathcal{M}_{\lambda}=\bigoplus_{j \in J}\left[\mathcal{F}^{2}\left(\mathcal{H}_{n}\right) \otimes \tilde{\varphi}_{j}\right]$ for some orthogonal inner functions $\varphi_{j}, j \in J$. Recall that $|J|$, the cardinality of $J$, is the dimension of the wandering subspace $\mathcal{L}_{\lambda}=\mathcal{M}_{\lambda} \ominus\left[S_{1} \mathcal{M}_{\lambda} \oplus \cdots \oplus S_{n} \mathcal{M}_{\lambda}\right]$ for $\left.S_{1}\right|_{\mathcal{M}_{\lambda}}, \cdots,\left.S_{n}\right|_{\mathcal{M}_{\lambda}}$. Moreover, whenever $\tilde{\varphi} \in \mathcal{L}_{\lambda}$ satisfies $\|\tilde{\varphi}\|_{2}=1$ we have that $\varphi$ is inner.

Let $Q_{\lambda}$ be the orthogonal projection onto $\mathcal{M}_{\lambda}$ and $P_{\lambda}$ the orthogonal projection onto $\mathcal{L}_{\lambda}$. One can easily check that $P_{\lambda}=Q_{\lambda}-\sum_{i \leq n} S_{i} Q_{\lambda} S_{i}^{*}$ and that

$$
\begin{aligned}
Q_{\lambda} e_{f} & =e_{f}-\frac{\lambda_{f}}{\left\|z_{\lambda}\right\|_{2}^{2}} z_{\lambda} \quad \text { for any } \quad f \in \mathcal{F}, \\
P_{\lambda} e_{i} & =\frac{1}{\left\|z_{\lambda}\right\|_{2}^{2}}\left(e_{i}-\lambda_{i}\right) \otimes z_{\lambda} \quad \text { for } \quad i \leq n, \\
P_{\lambda} e_{0} & =e_{0}-\frac{1}{\left\|z_{\lambda}\right\|_{2}^{2}} z_{\lambda} .
\end{aligned}
$$

If $e_{f}=e_{i} \otimes e_{g}$ for some $i \leq n$ and $g \in \mathcal{F}$ we can also check that $P_{\lambda} e_{f}=\frac{\lambda_{g}}{\left\|z_{\lambda}\right\|_{2}^{2}}\left(e_{i}-\right.$ $\left.\lambda_{i}\right) \otimes z_{\lambda}$

It is easy to see that (17) implies that $\tilde{z}_{\lambda}=z_{\lambda}$, since $\lambda_{f}=\lambda_{\tilde{f}}$. Then we get

Proposition 3.4. $\mathcal{L}_{\lambda}=\operatorname{span}\left\{\tilde{\varphi}_{0}, \tilde{\varphi}_{1}, \cdots, \tilde{\varphi}_{n}\right\}$ where

$$
\varphi_{0}=a_{0}\left[e_{0}-\frac{1}{\left\|z_{\lambda}\right\|_{2}^{2}} z_{\lambda}\right], \quad \text { and } \quad \varphi_{i}=a_{i}\left[z_{\lambda} \otimes\left(e_{i}-\lambda_{i}\right)\right] \quad i=1,2 \cdots, n
$$

The $a_{i} \in \mathbb{C}$ are chosen so that $\left\|\varphi_{i}\right\|_{2}=1$ for $i=0,1, \cdots, n$. Moreover, the $\varphi_{i}$ 's and any normalized linear combination of them are inner function.

Remarks. (1) We have that $\mathcal{M}_{\lambda}=\mathcal{F}^{2}\left(\mathcal{H}_{n}\right) \otimes \tilde{\varphi}_{0}+\mathcal{F}^{2}\left(\mathcal{H}_{n}\right) \otimes \tilde{\varphi}_{1}+\cdots+\mathcal{F}^{2}\left(\mathcal{H}_{n}\right) \otimes \tilde{\varphi}_{n}$. 
(2) We also have that $\psi \in \mathcal{M}_{\lambda}$ if and only if $\left\langle\psi, z_{\lambda}\right\rangle=0$ if and only if there exist $g_{i} \in \mathcal{F}^{2}\left(\mathcal{H}_{n}\right), i=0,1, \cdots, n$ such that

$$
\psi=g_{0} \otimes \tilde{\varphi}_{0}+g_{1} \otimes \tilde{\varphi}_{1}+\cdots+g_{n} \otimes \tilde{\varphi}_{n}
$$

This can be view as a "weak-factorization" result.

(3) It is easy to compute that $\left\|z_{\lambda}\right\|_{2}^{2}=\frac{1}{1-\|\lambda\|_{2}^{2}}$.

If $\mathcal{M}$ is an invariant subspace for $S_{1}, \cdots, S_{n}$ and $p \in \mathcal{P}$ we always have (Proposition 2.3) that $p \otimes \mathcal{M} \subset \mathcal{M}$. In general we do not have that $\mathcal{M} \otimes p \subset \mathcal{M}$. However, this is always true for the 1-codimensional invariant subspaces.

Lemma 3.5. Let $\mathcal{M}_{\lambda}=\left[z_{\lambda}\right]^{\perp}$ for some $\lambda \in \mathbb{C}^{n},\|\lambda\|_{2}<1$. For any $p \in \mathcal{P}$ we have that $p \otimes \mathcal{M}_{\lambda} \subset \mathcal{M}_{\lambda}$ and $\mathcal{M}_{\lambda} \otimes p \subset \mathcal{M}_{\lambda}$.

Proof. The first inclusion is clear. If $\varphi \in \mathcal{M}_{\lambda}$ and $p \in \mathcal{P}$ then $\left\langle p \otimes \varphi, z_{\lambda}\right\rangle=0$.

The key point for the other one is that $\varphi \in \mathcal{M}_{\lambda}$ if and only if $\tilde{\varphi} \in \mathcal{M}_{\lambda}$. To see this recall that $z_{\lambda}=\tilde{z}_{\lambda}$. Then

$$
\left\langle\tilde{\varphi}, z_{\lambda}\right\rangle=\left\langle\varphi, \tilde{z}_{\lambda}\right\rangle=\left\langle\varphi, z_{\lambda}\right\rangle
$$

If $p \in \mathcal{P}$ and $\varphi \in \mathcal{M}_{\lambda}$ we have that

$$
\left\langle\varphi \otimes p, z_{\lambda}\right\rangle=\left\langle\varphi \otimes p, \tilde{z}_{\lambda}\right\rangle=\left\langle\widetilde{\varphi \otimes p}, z_{\lambda}\right\rangle=\left\langle\tilde{p} \otimes \tilde{\varphi}, z_{\lambda}\right\rangle=0 .
$$

Let $\mathcal{N}=\bigcap_{\|\lambda\|_{2}<1} \mathcal{M}_{\lambda}$ and $\mathcal{N}^{\infty}=\mathcal{N} \cap F^{\infty} . \mathcal{N}$ is a "2-sided" invariant subspace and $\mathcal{N}^{\infty}$ is a two-sided ideal in $F^{\infty}$.

There are plenty of elements in $\mathcal{N}$. For example $\varphi=e_{1} \otimes e_{2}-e_{2} \otimes e_{1} \in \mathcal{N}$. For every $\lambda \in \mathbb{C}^{n},\|\lambda\|_{2}<1$ we have

$$
\left\langle\varphi, z_{\lambda}\right\rangle=\left\langle e_{1} \otimes e_{2}, z_{\lambda}\right\rangle-\left\langle e_{2} \otimes e_{1}, z_{\lambda}\right\rangle=\lambda_{1} \lambda_{2}-\lambda_{2} \lambda_{1}=0
$$

More generally if $f \in F(k, \Lambda)$ and $\pi \in \Pi_{k},(\pi$ is a permutation on $\{1,2, \cdots, k\})$, let $\pi(f) \in F(k, \Lambda)$ be defined by $\pi(f)(i)=f(\pi(i))$. Then $e_{f}-e_{\pi(f)} \in \mathcal{N}$.

Proposition 3.6. $F^{\infty} / \mathcal{N}^{\infty}$ is a commutative algebra.

Proof. Let $\varphi=\sum_{f \in \mathcal{F}} a_{f} e_{f}, \psi=\sum_{g \in \mathcal{F}} b_{g} e_{g}$ be elements in $F^{\infty}$. We want to prove that $\varphi \otimes \psi-\psi \otimes \varphi \in \mathcal{N}$. Notice that

$$
\varphi \otimes \psi-\psi \otimes \varphi=\sum_{f \in \mathcal{F}} \sum_{g \in \mathcal{F}} a_{f} b_{g}\left[e_{f} \otimes e_{g}-e_{g} \otimes e_{f}\right] .
$$

Let $\lambda \in \mathbb{C}^{n},\|\lambda\|_{2}<1$. Since $\left\langle e_{f} \otimes e_{g}-e_{g} \otimes e_{f}, z_{\lambda}\right\rangle=0$ we get that $\langle\varphi \otimes \psi-\psi \otimes$ 


\section{Reflexivity RESUlts}

Let $\mathcal{H}$ be a Hilbert space and $B(\mathcal{H})$ be the algebra of all bounded operators on $\mathcal{H}$. If $A \in B(\mathcal{H})$ then the set of all invariant subspaces of $A$ is denoted by Lat $A$. For any $\mathcal{U} \subset B(\mathcal{H})$ we define

$$
\text { Lat } \mathcal{U}=\bigcap_{A \in \mathcal{U}} \text { Lat } A \text {. }
$$

If $\mathcal{S}$ is any collection of subspaces of $\mathcal{H}$, then $\operatorname{Alg} \mathcal{S}:=\{A \in B(\mathcal{H}): \mathcal{S} \subset$ Lat $A$ \}. The algebra $\mathcal{U} \subset B(\mathcal{H})$ is reflexive if $\mathcal{U}=\operatorname{Alg}$ Lat $\mathcal{U}$.

The main theorems of this section are the following.

Theorem 4.1. The algebra $\left\{\varphi\left(S_{1}, S_{2}, \cdots, S_{n}\right): \varphi \in F^{\infty}\right\}$ is reflexive.

Theorem 4.2. If $\mathcal{U}$ is a strongly closed subalgebra of $\left\{\varphi\left(S_{1}, \cdots, S_{n}\right) ; \varphi \in F^{\infty}\right\}$ containing the identity then $\mathcal{U}$ is reflexive.

Proof of Theorem 4.1. Let $A \in \operatorname{Alg} \operatorname{Lat} F^{\infty}$. For every $\varphi$ inner we have that $F^{2}\left(H_{n}\right) \otimes \tilde{\varphi} \in$ Lat $F^{\infty}$. Then $A\left[F^{2}\left(H_{n}\right) \otimes \tilde{\varphi}\right] \subset F^{2}\left(H_{n}\right) \otimes \tilde{\varphi}$. In particular we have

$$
A \tilde{\varphi}=\psi_{\varphi} \otimes \tilde{\varphi} \quad \text { for some } \quad \psi_{\varphi} \in F^{2}\left(H_{n}\right)
$$

For every $f \in \mathcal{F}$ we can find $\psi_{f}$ such that

$$
A e_{f}=\psi_{f} \otimes e_{f} .
$$

(Notice that both $e_{f}$ and $\tilde{e}_{f}$ are inner and we do not have to carry the tilde).

Let $k \geq 1$. Example 4 of Section 3 tells that $x_{k}=a_{k} \sum_{f \in F(k, \Lambda)} e_{f}$, where $a_{k}=[\operatorname{card}(F(k, \Lambda))]^{-1 / 2}$, is inner (notice that $x_{k}=\tilde{x}_{k}$ ). Using (18) we have that

$$
A x_{k}=\psi_{k} \otimes x_{k}=a_{k} \sum_{f \in F(k, \Lambda)} \psi_{k} \otimes e_{f} .
$$

On the other hand, we have that

$$
A x_{k}=A\left(a_{k} \sum_{f \in F(k, \Lambda)} e_{f}\right)=a_{k} \sum_{f \in F(k, \Lambda)} A e_{f}=a_{k} \sum_{f \in F(k, \Lambda)} \psi_{f} \otimes e_{f} .
$$

We claim that for every $f \in F(k, \Lambda), \psi_{k}=\psi_{f}$. Fix $f \in F(k, \Lambda)$ and let $P_{f}$ be the orthogonal projection onto $\mathcal{F}^{2}\left(\mathcal{H}_{n}\right) \otimes e_{f}$. From (19) we get that $P_{f}\left(A x_{k}\right)=$ $a_{k} \psi_{k} \otimes e_{f}$ (the other terms are zero), and from (20) we get that $P_{f}\left(A x_{k}\right)=a_{k} \psi_{f} \otimes e_{f}$. 
Let $k>1$. Example 5 of Section 3 tells us that $y=\frac{1}{\sqrt{2}}\left[e_{1}+e_{2}^{k}\right]$ is inner (notice that $y=\tilde{y})$. Using (18) we have that

$$
A y=\psi_{y} \otimes y=\frac{1}{\sqrt{2}}\left[\psi_{y} \otimes e_{1}+\psi_{y} \otimes e_{2}^{k}\right]
$$

On the other hand,

$$
A y=\frac{1}{\sqrt{2}}\left[A e_{1}+A e_{2}^{k}\right]=\frac{1}{\sqrt{2}}\left[\psi_{1} \otimes e_{1}+\psi_{k} \otimes e_{2}^{k}\right]
$$

The last equality is clear since $e_{1}$ has one letter and $e_{2}^{k}$ has $k$ letters. Combining (21) and (22) we conclude that $\psi_{k}=\psi_{1}$.

Summarizing we have that $A e_{0}=\psi_{0}$ for some $\psi_{0} \in F^{2}\left(H_{n}\right)$, and if $f \in F(k, \Lambda)$, $k \geq 1$, then $A e_{f}=\psi_{1} \otimes e_{f}$. It is easy to see that $\psi_{1} \in F^{\infty}$.

Let $B=A-\psi_{1}\left(S_{1}, S_{2}, \cdots, S_{n}\right)$. We still have that $B \in \operatorname{Alg}$ Lat $F^{\infty}$ and if we set $\psi=\psi_{0}-\psi_{1}$

$$
\begin{aligned}
& B e_{0}=\psi \\
& B e_{f}=0 \quad \text { if } \quad f \neq 0 .
\end{aligned}
$$

We want to prove that $B=0$. This gives us that $A=\psi_{1} \in F^{\infty}$.

Equation (18) applies to $B$ as well. That is, if $\varphi$ is inner, then $B \tilde{\varphi}=\psi_{\varphi} \otimes \tilde{\varphi}$. On the other hand it is clear that $B \tilde{\varphi}=\left\langle e_{0}, \tilde{\varphi}\right\rangle \psi$. Hence, if $\left\langle e_{0}, \tilde{\varphi}\right\rangle \neq 0$, we have that

$$
\psi=\frac{1}{\left\langle e_{0}, \tilde{\varphi}\right\rangle} \psi_{\varphi} \otimes \tilde{\varphi} \in F^{2}\left(H_{n}\right) \otimes \tilde{\varphi}, \quad \text { and } \quad \tilde{\psi} \in \varphi \otimes \mathcal{F}^{2}\left(\mathcal{H}_{n}\right) \text {. }
$$

It is easy to see that $\varphi(f, \mu)$ (the Möbius maps of Example 7, Section 3) satisfy $\left\langle e_{0}, \tilde{\varphi}(f, \mu)\right\rangle \neq 0$ for any $f \in \mathcal{F}$ and $0<|\mu|<1$. This implies that

$$
\tilde{\psi} \in \bigcap\left\{\varphi(f, \mu) \otimes \mathcal{F}^{2}\left(\mathcal{H}_{n}\right): f \in \mathcal{F}, 0<|\mu|<1\right\}
$$

By Lemma 3.2 we conclude that $\psi=0$.

Remark The previous proof does not work in the commutative case. We are using the fact that $e_{1}$ and $e_{2}$ are non-commutative to conclude that $\frac{1}{\sqrt{2}}\left[e_{1}+e_{2}^{k}\right]$ is inner. However, $\frac{1}{\sqrt{2}}\left[z+z^{k}\right]$ is not inner in $H^{\infty}$.

The following notation will be useful in the proof of Theorem 4.2: If $A \in B(\mathcal{H})$ and $m$ is a positive integer, then $\mathcal{H}^{(m)}$ denotes the direct sum of $m$ copies of $\mathcal{H}$ and $A^{(m)}$ stands for the direct sum of $m$ copies of $A$. If $\mathcal{U} \subset B(\mathcal{H})$, then 
According to [RR, Theorem 7.1], if $\mathcal{U}$ is an algebra of operators containing the identity, then the closure of $\mathcal{U}$ in the strong operator topology is

$$
\left\{B: \text { Lat } \mathcal{U}^{(m)} \subset \text { Lat } B^{(m)} \text { for } m=1,2,3, \cdots\right\}
$$

To prove Theorem 4.2 we need the following

Theorem 4.3. If $A, B \in\left\{\varphi\left(S_{1}, \cdots, S_{n}\right) ; \quad \varphi \in F^{\infty}\right\}$ are such that Lat $A \subset$ Lat $B$, then

$$
\text { Lat } A^{(m)} \subset \text { Lat } B^{(m)}, \quad \text { for any } m=1,2, \cdots \text {. }
$$

Proof. It will enough to show that every cyclic invariant subspace of $A^{(m)}$ is invariant under $B^{(m)}$. Let $x \neq 0$ be an element in $F^{2}\left(H_{n}\right)^{(m)}$ and let $\mathcal{M}=$ $\bigvee_{f \in \mathcal{F}} S_{f}^{(m)} x$. According to Lemma 2.5 there is a unitary operator $U: F^{2}\left(H_{n}\right) \rightarrow \mathcal{M}$ such that

$$
\left.S_{i}^{(m)}\right|_{\mathcal{M}}=U S_{i} U^{-1}, \quad \text { for any } i=1,2, \cdots
$$

Since $A$ and $B$ are strong limits of polynomials in $S_{1}, \cdots, S_{n}$ it follows that

$$
\begin{aligned}
& \left.A^{(m)}\right|_{\mathcal{M}}=U A U^{-1}, \quad \text { and } \\
& \left.B^{(m)}\right|_{\mathcal{M}}=U B U^{-1} .
\end{aligned}
$$

Let $\mathcal{G}$ be the smallest invariant subspace of $A^{(m)}$ containing $x$, i.e.,

$$
\mathcal{G}=\bigvee_{f \in \mathcal{F}} A_{f}^{(m)} x
$$

Since $A^{(m)}=\varphi\left(S_{1}^{(m)}, \cdots, S_{n}^{(m)}\right)$ for some $\varphi \in F^{\infty}$ it follows that $\mathcal{G} \subset \mathcal{M}$. The relation $(23)$ and the fact that $A^{(m)} \mathcal{G} \subset \mathcal{G}$ implies $U A U^{-1}(\mathcal{G}) \subset \mathcal{G}$ whence $A\left(U^{-1} \mathcal{G}\right) \subset U^{-1} \mathcal{G}$. But Lat $A \subset$ Lat $B$ implies $B\left(U^{-1} \mathcal{G}\right) \subset U^{-1} \mathcal{G}$. Therefore, $U B U^{-1}(\mathcal{G}) \subset \mathcal{G}$ which together with $(23)$ show that $B^{(m)} \mathcal{G} \subset \mathcal{G}$.

Corollary 4.4. Let $A, B \in\left\{\varphi\left(S_{1}, \cdots, S_{n}\right): \varphi \in F^{\infty}\right\}$ be such that Lat $A \subset$ Lat $B$, then $B$ belongs to the strongly closed algebra generated by $A$ and the identity.

The proof of the following theorem is analogue to the proof of the Theorem 4.3 if we replace $A$ with any subset $\mathcal{A} \subset\left\{\varphi\left(S_{1}, \cdots, S_{n}\right): \quad \varphi \in F^{\infty}\right\}$. We omit the 
Theorem 4.5. If $B \in\left\{\varphi\left(S_{1}, \cdots, S_{n}\right): \varphi \in F^{\infty}\right\}$ is such that Lat $\mathcal{A} \subset$ Lat $B$ then

$$
\text { Lat } \mathcal{A}^{(m)} \subset \text { Lat } B^{(m)}, \quad \text { for any } m=1,2, \cdots \text {. }
$$

Moreover, $B$ belongs to the strongly closed algebra generated by $\mathcal{A}$ and the identity.

Proof of Theorem 4.1. Let $B \in B\left(\mathcal{F}^{2}\left(\mathcal{H}_{n}\right)\right)$ be such that

$$
\text { Lat } \mathcal{U} \subset \text { Lat } B \text {. }
$$

It is clear that

$$
\text { Lat }\left\{S_{1}, \cdots, S_{n}\right\} \subset \text { Lat }\left\{\varphi\left(S_{1}, \cdots, S_{n}\right) ; \quad \varphi \in F^{\infty}\right\} \subset \text { Lat } \mathcal{U} \subset \text { Lat } B \text {. }
$$

According to Theorem 4.1, we have

$$
B \in\left\{\varphi\left(S_{1}, \cdots, S_{n}\right) ; \quad \varphi \in F^{\infty}\right\}
$$

Now Theorem 4.5 implies that $B \in \mathcal{U}$.

Let us recall from [Po2] that an operator $T \in B\left(\mathcal{F}^{2}\left(\mathcal{H}_{n}\right)\right)$ is called multi-analytic if $T S_{i}=S_{i} T$ for each $i \in \Lambda=\{1,2, \cdots, n\}$. The following result is an easy consequence of Theorem 4.2 and the characterization of the multi-analytic operators in terms of their symbols [Po4].

Corollary 4.6. Any strongly closed subalgebra of multi-analytic operators containing the identity is reflexive.

Let us remark that in the particular case when $\Lambda=\{1\}$ we find again the result of Sarason $[\mathrm{S}]$.

\section{Open Questions}

We finish this paper with some questions.

Let Inv $\left(F^{\infty}\right)$ be the group of invertible elements in $F^{\infty}$, and $\mathcal{G}_{0}$ be the connected component in Inv $\left(F^{\infty}\right)$ which contains the identity. From the general theory of Banach algebras [D] we know that the collection of finite products of elements in 
Problem 1. Characterize Inv $\left(F^{\infty}\right) / \mathcal{G}_{0}$.

Problem 2. Characterize the proper maximal invariant subspaces for the $\Lambda$-orthogonal shift of $\mathcal{F}^{2}\left(\mathcal{H}_{n}\right)$.

It is clear that all of the $\mathcal{M}_{\lambda}$ of Example 8, Section 3 are maximal. However, there are many invariant subspaces for the shift that are not inside any of them.

Take, for instance, $e_{f}=e_{1} \otimes e_{2} \otimes \cdots \otimes e_{n}$ and $\mu \in \mathbb{C}, \frac{1}{\sqrt{n}}<|\mu|<1$. We claim that $\mathcal{M}=\mathcal{F}^{2}\left(\mathcal{H}_{n}\right) \otimes \tilde{\varphi}(f, \mu)$ is not a subset of $\mathcal{M}_{\lambda}$ for any $\lambda \in \mathbb{C}^{n},\|\lambda\|_{2}<1$. (See Example 6, Section 3 for the notation).

It is clear that $\mathcal{M} \subset \mathcal{M}_{\lambda}$ if and only if $z_{\lambda} \in \mathcal{M}^{\perp}$ and since $z_{\lambda}=\tilde{z}_{\lambda}$ it is equivalent to $z_{\lambda} \in\left[\varphi(f, \mu) \otimes \mathcal{F}^{2}\left(\mathcal{H}_{n}\right)\right]^{\perp}$. Using (16) we get that $\mathcal{M} \subset \mathcal{M}_{\lambda}$ if and only if

$$
\begin{aligned}
& \forall g \in \mathcal{F}, \quad\left\langle e_{f} \otimes e_{g}\right\rangle=\mu\left\langle e_{g}, z_{\lambda}\right\rangle, \quad \text { equivalently } \\
& \forall g \in \mathcal{F}, \quad \lambda_{f} \lambda_{g}=\mu \lambda_{g} .
\end{aligned}
$$

Since $\lambda_{0}=1$, we have that $\lambda_{f}=\lambda_{1} \lambda_{2} \cdots \lambda_{n}=\mu$. However, $\sum_{i \leq n}\left|\lambda_{i}\right|^{n}<1$ implies that

$$
|\mu|=\left|\lambda_{1} \lambda_{2} \cdots \lambda_{n}\right|<\frac{1}{\sqrt{n}}
$$

contradicting the condition on $\mu$.

Problem 3. Characterize the proper maximal subspaces of $\mathcal{F}^{2}\left(\mathcal{H}_{n}\right)$ of the form $\varphi \otimes \mathcal{F}^{2}\left(\mathcal{H}_{n}\right), \varphi$ inner.

It follows from Corollary 2.7 that if $\varphi_{1}, \varphi_{2}$ are inner and $\varphi_{1} \otimes \mathcal{F}^{2}\left(\mathcal{H}_{n}\right) \subset \varphi_{2} \otimes$ $\mathcal{F}^{2}\left(\mathcal{H}_{n}\right)$ then there exists $\varphi_{3}$ inner such that $\varphi_{1}=\varphi_{2} \otimes \varphi_{3}$. Then the maximal subspaces of the form $\varphi \otimes \mathcal{F}^{2}\left(\mathcal{H}_{n}\right)$ correspond to "prime" inner functions.

Problem 4. Is every inner function $\varphi$ a product of "prime" ones? The question referes mainly to convergence.

\section{REFERENCES}

[A] A. Arias, Isomorphisms of operator algebras, preprint.

[D] R. G. Douglas, Banach algebra techniques in operator theory, Academic Press, New York (1972).

[Po1] G. Popescu, Characteristic functions for infinite sequences of noncommuting operators, J. Operator Theory 22 (1989), 51-71.

[Po2] _ Multi-analytic operators and some factorization theorems, Indiana Univ. Math. J. 38 (1989), 693-710.

[Po3] _ Von Neumann inequality for $\left(B(H)^{n}\right)_{1}$, Math.Scand. 68 (1991), 292-304. 
[RR] H. Radjavi, P. Rosental, Invariant subspaces, Springer-Verlag, Berlin (1973).

[S] D. Sarason, Invariant subspaces and unstarred operator algebras, Pacific J.Math. 17 (1966), 511-517.

[vN] J. von Neumann, Eine Spectraltheorie für allgemeine Operatoren eines unitären Raumes, Math. Nachr. 4 (1951), 258-281.

\section{A. ARIAS}

Division of Mathematics, Computer Science and Statistics, The University of Texas At San Antonio, SAn Antonio, TX 78249, U.S.A.

E-mail address: arias@ringer.cs.utsa.edu

G. Popescu

Division of Mathematics, Computer Science and Statistics, The University of Texas at San Antonio, San Antonio, TX 78249, U.S.A.

E-mail address: gpopescu@ringer.cs.utsa.edu 